\title{
分体圆柱谐振腔法用于金刚石膜微波介电性能测试的研究
}

\author{
苏静杰 ${ }^{1,2}$, 杨 梓 $^{1}$, 李义锋 ${ }^{1}$, 唐伟忠 ${ }^{1}$, 安晓明 ${ }^{3}$, 郭 辉 ${ }^{3}$
}

(1. 北京科技大学 材料科学与工程学院, 北京 100083 ; 2. 中国科学院 北京纳米能源与系统研究所, 北京 100083 ; 3.河北省科学院 激光研究所, 石家庄 050081)

摘 要: 针对金刚石膜微波介电损耗低、厚度薄带来的微波介电性能测试难点, 研制了一台分体圆柱谐振腔式微波 介电性能测试装置。利用不同直径的蓝宝石单晶样品, 用上述装置对低损耗薄膜类样品微波介电性能的测试能力及 样品直径对测试结果的影响进行了实验研究。在此基础上，使用分体圆柱谐振腔式微波介电性能测试装置对微波等 离子体化学气相沉积法和直流电弧等离子体喷射法制备的高品质金刚石膜在 $\mathrm{Ka}$ 波段的微波介电性能进行了测试 比较。测试结果表明，由 Raman 光谱、紫外-可见光谱等分析证明品质较优的微波等离子体化学气相沉积法制备的 金刚石膜具有更高的微波介电性能, 其相对介电常数和微波介电损耗值均低于直流电弧等离子体喷射法制备的金 刚石膜。

关 键 词: 微波介电性能; 分体圆柱谐振腔; 金刚石膜

中图分类号: TQ174 文献标识码: A

\section{Measurement of Microwave Dielectric Properties of Diamond Films Using Split-cylinder Resonator Method}

\begin{abstract}
SU Jing-Jie ${ }^{1,2}$, YANG Zi ${ }^{1}$, LI Yi-Feng ${ }^{1}$, TANG Wei-Zhong ${ }^{1}$, AN Xiao-Ming ${ }^{3}$, GUO Hui ${ }^{3}$
(1. School of Materials Science and Engineering, University of Science and Technology Beijing, Beijing 100083, China; 2. Beijing Institute of Nanoenergy and Nanosystems, Chinese Academy of Science, Beijing 100083, China; 3. Institute of Hebei Laser Technology, Shijiazhuang 050081, China)

Abstract: Regarding to the difficulties in measuring microwave dielectric properties of diamond films due to their characteristics of low dielectric loss and small thickness, a split-cylinder resonator apparatus was established. By measuring dielectric performances of sapphire samples with different diameters, the capability of the split-cylinder resonator for measuring low dielectric loss materials was demonstrated, and the influence of sample's diameter on measurement results was studied. Then by using the split-cylinder resonator apparatus, dielectric properties of high quality diamond films prepared by two different methods, microwave plasma chemical vapor deposition (MPCVD) and DC arc plasma jet, were measured in the Ka band. Results show that the diamond film deposited by MPCVD method has a higher quality than that of the diamond film deposited by the DC arc plasma jet method, which is consistent with the results of their Raman and UV-visible absorption spectra. The results indicate that both the relative dielectric constant and the loss tangent of the sample deposited by MPCVD method are lower than those of the sample prepared by DC arc plasma jet method.
\end{abstract}

Key words: dielectric property; split-cylinder resonator; diamond film

近年来, 随着微波电真空器件向着大功率、宽 频带、小型化和高频率的方向发展，对微波介电材
料的性能提出了更高的要求 ${ }^{[1-2]}$ 。研究表明, 用化学 气相沉积方法制备的金刚石膜材料拥有微波介电损 
耗低和热导率高等优异特性, 是一种理想的微波介 电材料。为了实现金刚石膜在微波电真空器件领域 中的应用, 对金刚石膜的微波介电性能开展系统的 测试研究十分必要。

虽然人们已经发展了多种材料微波介电性能测 试方法，但对于金刚石膜而言，其微波损耗低和厚 度薄的特点使其微波介电性能的准确测量有一定难 度。目前, 国际上有关金刚石膜介电性能测试结果 的报道很少，并且多以金刚石膜应用于核聚变堆高 功率回旋管微波窗口为背景展开的, 因而测试的频 率集中在 140 170 GHz 范围内 ${ }^{[3-10]}$ 。而国内关于金 刚石膜微波介电性能的研究更少, 且仅局限于低于 $10 \mathrm{GHz}$ 的低频率段 ${ }^{[11-12]}$ 。对于金刚石膜在毫米波雷 达所使用的重要频段之一的 $\mathrm{Ka}$ 波段(27 40 GHz)中 微波介电性能的研究尚未见到报道。

在众多的介电性能测试方法中，谐振腔法是低 损耗样品的介电性能最为准确的测试方法。而谐振 腔法又分为很多种, 如分体圆柱式谐振腔法、分体 介质柱式谐振腔法、平行板介质柱式谐振腔法和回 音壁式谐振腔法等, 其中, 由 Janezic 等 ${ }^{[13]}$ 提出的分 体圆柱式谐振腔法适用于低损耗和厚度较小的薄膜 类样品。针对目前金刚石膜微波介电性能测试的困 难及其在 $\mathrm{Ka}$ 波段介电性能测试数据的空缺, 本工 作尝试建立一套分体圆柱谐振腔式材料微波介电性 能测试装置, 并将其应用于高品质金刚石膜样品在 $\mathrm{Ka}$ 波段的微波介电性能测量。

\section{1 分体圆柱谐振腔式微波介电性能测 试装置}

图 1 为分体圆柱谐振腔式微波介电性能测试装 置的结构示意图, 从图中可以看出, 分体圆柱谐振

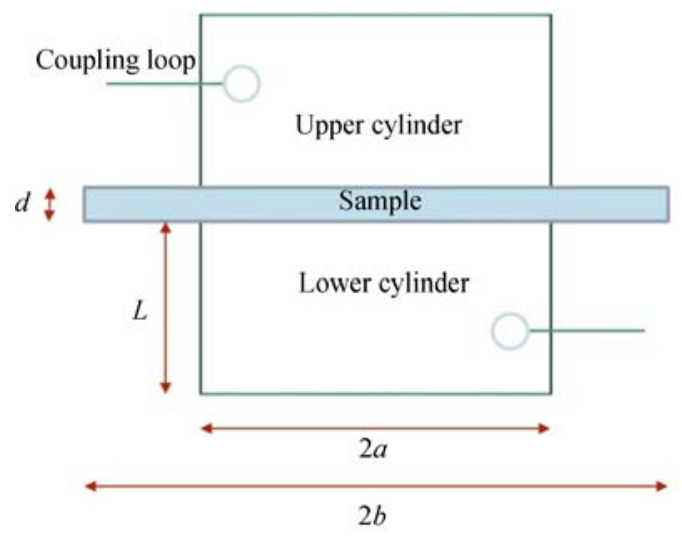

图 1 分体圆柱谐振腔式微波介电性能测试装置的示意图

Fig. 1 Schematic of the split-cylinder resonator for dielectric property measurement
腔由两个对称的圆柱形半谐振腔组成, 待测的片状 样品放在上下两个半谐振腔的结合处。在每个圆柱 形半谐振腔的中部都开有一个耦合孔，微波信号通 过同轴耦合的方式被送入谐振腔中。待测样品的直 径为 $2 b$, 厚度为 $d$, 而半谐振腔的直径为 $2 a$, 高度 为 $L$ 。

针对 $\mathrm{Ka}$ 波段的测试需求，分体圆柱谐振腔的 尺寸被设定为 $2 a=13.18 \mathrm{~mm}, L=3.51 \mathrm{~mm}$ 。根据公式 (1), 可计算出分体圆柱谐振腔 $\mathrm{TE}_{011}$ 模式的空腔谐 振频率 $f_{011}$ 为 $35 \mathrm{GHz}$, 它正好处于 $\mathrm{Ka}$ 波段频率范 围内。

$$
f_{011}=\frac{c}{2 \pi \sqrt{\varepsilon_{a}^{\prime}}} \sqrt{\left(\frac{j_{1}}{a}\right)^{2}+\left(\frac{\pi}{2 L}\right)^{2}}
$$

在式(1)中, $\mathrm{c}$ 为真空中的光速, $\varepsilon_{a}^{\prime}$ 为空气的相对介电常 数, $j_{1}$ 为第一类一阶贝塞尔函数的第一个零点值。

图 2 为所研制的分体圆柱谐振腔式微波介电性 能测试装置的实物图, 它选用了导电性较好的无氧 铜材料来制造，并对其表面进行了镀金处理。测试 过程中, 经同轴线耦合进谐振腔内的微波将形成 $\mathrm{TE}_{011}$ 模式的谐振。为了方便调节并使谐振腔处于弱 耦合状态，在谐振腔上设计了直进式的调节机构。 将谐振腔与安捷伦 E5244A 矢量网络分析仪相连接, 即可测量不同频率下微波通过谐振腔时的传输损 耗。对微波传输损耗随频率的变化曲线进行拟合和 计算，即可得到待测样品的相对介电常数和微波介 电损耗角正切值 ${ }^{[14]}$ 。

\section{2 分体圆柱谐振腔式微波介电性能测 试装置的可靠性验证}

为了了解分体圆柱谐振腔式微波介电性能测试 装置的测试能力，选用四个不同直径的蓝宝石单晶 样品对装置测试结果的可靠性进行验证。四个蓝宝

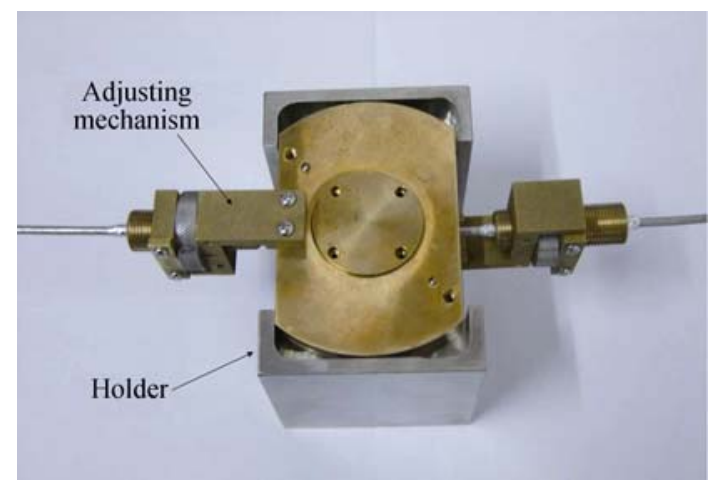

图 2 分体圆柱谐振腔式微波介电性能测试装置的实物图 Fig. 2 Photograph of the split-cylinder resonator for dielectric properties measurement 
石单晶样品来自同一批次，直径分别为 $\phi 18.2$ 、 $\phi 21.6 、 \phi 25$ 和 $\phi 30 \mathrm{~mm}$, 厚度均为 $(335 \pm 5) \mu \mathrm{m}$, 单晶 的取向为 $<0001>$ 方向。由于在分体圆柱谐振腔式微 波介电性能测试装置中, 微波电场的谐振模式为 $\mathrm{TE}_{011}$ 模式, 因而对于具有六方结构、取向为 $<0001>$ 方向的蓝宝石单晶样品而言, 测量出的将是样品垂 直于 $c$ 轴方向的微波介电性能。

表 1 是由分体圆柱谐振腔式测试装置测出的蓝宝 石单晶样品的微波介电性能, 包括相对介电常数 $\varepsilon_{r}^{\prime}$ 和 微波介电损耗角正切值 $\tan \delta$ 。从表 1 可以看出, 四个蓝 宝石单晶样品的相对介电常数和微波介电损耗角正切 值的测试结果彼此间差异很小, 分别只有 0.05 和 $10^{-6}$ 数量级, 即不同样品测试结果间的差异均处于测试误 差范围内。这表明, 当样品的直径> $18.2 \mathrm{~mm}$ 时, 样品 直径对其材料微波介电性能的测试结果无影响。这一 结果为金刚石膜样品尺寸的选择提供了依据。

从表 1 还可以看出, 四个蓝宝石单晶样品的介 电常数测量值均处于 9.40 9.45 之间, 与文献报道 的室温下蓝宝石单晶垂直于 $c$ 轴方向的介电常数值 9.40 十分接近 ${ }^{[15]}$ 。这一结果证明, 分体圆柱谐振腔 式微波介电性能测试装置具有准确测量低损耗薄膜 样品的相对介电常数的能力。与此同时, 四个蓝宝 石单晶样品的微波介电损耗角正切值均处于 $(0.26 \sim 0.27) \times 10^{-4}$ 之间, 这符合高品质的蓝宝石单晶 低微波介电损耗的特点, 并且四个样品测试结果之 间的差异小于测试误差, 又证明分体圆柱谐振腔式 微波介电性能测试装置具有较高的测试稳定性。

上述对于不同直径的蓝宝石单晶样品的测试, 既考察了样品直径对样品微波介电性能测试结果的

表 1 不同直径蓝宝石单晶样品在垂直于 $\mathrm{c}$ 轴方向的微波介 电性能

Table 1 Microwave dielectric properties of sapphire samples ( $\perp$ c axis) with different diameter

\begin{tabular}{cccc}
\hline $\begin{array}{c}\text { Diameter } \\
\mathrm{mm}\end{array}$ & $\begin{array}{c}\text { Frequency } / \\
\mathrm{GHz}\end{array}$ & $\varepsilon_{\mathrm{r}}^{\prime}$ & $\tan \delta / \times 10^{-4}$ \\
\hline 18.2 & 24.287 & $9.45 \pm 0.09$ & $0.26 \pm 0.20$ \\
21.6 & 24.399 & $9.45 \pm 0.09$ & $0.27 \pm 0.20$ \\
25.0 & 24.451 & $9.40 \pm 0.09$ & $0.27 \pm 0.20$ \\
30.0 & 24.476 & $9.44 \pm 0.09$ & $0.27 \pm 0.20$ \\
\hline
\end{tabular}

影响，又完成了对分体圆柱谐振腔式微波介电性能 测试装置测试结果可靠性的验证。

\section{3 高品质金刚石膜微波介电性能的测试}

采用所研制的分体圆柱谐振腔式微波介电性 能测试装置对两种不同方法制备的高品质金刚石 膜进行了微波介电性能测试。微波等离子体化学 气相沉积 $(M P C V D)$ 法 $^{[16]}$ 和直流电弧等离子体喷 射(DC Arc Plasma Jet)法 ${ }^{[17]}$ 是两种常用的金刚石 膜制备方法，两种方法都可以制备较高品质的金 刚石膜, 但它们分别具有纯净度高和生长速率快 的特点。

图 3 是采用 MPCVD 和 DC Arc Plasma Jet 两种 方法制备的双面抛光的高品质金刚石膜样品的照片, 其中图 3(a)为采用研究组新研制的穹顶谐振腔式高 功率 MPCVD 金刚石膜沉积装置 ${ }^{[18]}$ 制备的金刚石膜, 而图 3(b)为采用高功率 DC Arc Plasma Jet 方法制备 的金刚石膜。样品的直径均为 $18.2 \mathrm{~mm}$, 厚度分别 为 $277 \mu \mathrm{m}$ 和 $213 \mu \mathrm{m}$ 。两种金刚石膜的制备条件如 表 2 所示。

通过图 3 可以看出, 在宏观上, MPCVD 法制备 的金刚石膜呈现为无色透明状, 而 DC Arc Plasma Jet 法制备的金刚石膜则呈现为微黄色透明状。由于 金刚石膜的颜色与其内部含有的杂质和缺陷密度有 关，因而图 3 中两个样品外观上的差异表明, MPCVD 法制备的金刚石膜相比于 DC Arc Plasma Jet 法制备的金刚石膜有着更高的品质。

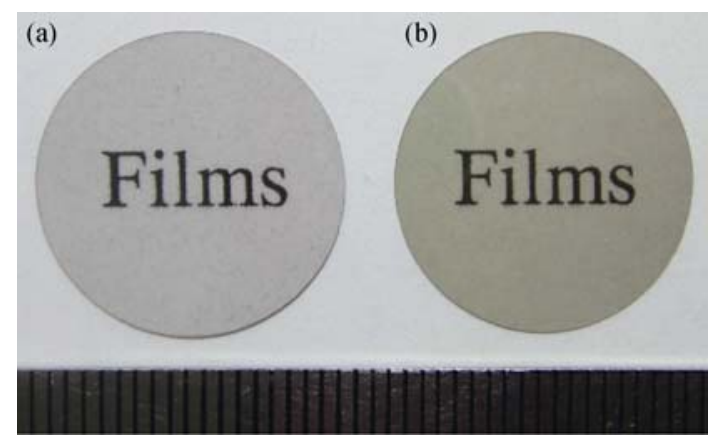

图 3 (a) MPCVD 法和(b) DC Arc Plasma Jet 法制备的金刚石 膜样品的照片

Fig. 3 Photos of diamond films deposited by (a) MPCVD and (b) DC Arc Plasma Jet methods

表 2 MPCVD 和 DC Arc Plasma Jet 法制备高品质金刚石膜样品的工艺条件

Table 2 Deposition conditions of the high quality diamond film prepared by two different methods

\begin{tabular}{ccccc}
\hline Method & Power $/ \mathrm{kW}$ & Pressure $/ \mathrm{kPa}$ & Flow rate $/ \mathrm{sccm}$ & Temperature $/{ }^{\circ} \mathrm{C}$ \\
\hline MPCVD & 9.2 & 15.8 & $\begin{array}{c}\mathrm{CH}_{4} / \mathrm{H}_{2}: 7 / 200 \\
\mathrm{CH}_{4} / \mathrm{H}_{2} / \mathrm{Ar}: 0.015 / 6 / 2\end{array}$ & 1030 \\
DC Arc Plasma Jet & 13.5 & 3.5 & in gas recycling mode) & 1000 \\
\hline
\end{tabular}


图 4 是上述两种金刚石膜样品的激光 Raman 光 谱, 由图可以看到, MPCVD 法制备的金刚石特征峰 半高宽 $(\mathrm{FWHM})$ 为 $2.0 \mathrm{~cm}^{-1}$, 接近于天然 IIa 型金刚 石膜的半高宽; DC Arc Plasma Jet 法制备的金刚石 膜特征峰半高宽为 $3.5 \mathrm{~cm}^{-1}$, 明显大于前者。另外, MPCVD 法制备金刚石膜的 Raman 谱中除了尖锐的 金刚石特征峰外, 不存在其他非金刚石相的杂质峰, 且曲线的背底很弱; 而 DC Arc Plasma Jet 法制备金 刚石膜的 Raman 谱中虽然也不存在明显的非金刚 石相杂质峰, 但其背底却较为明显。由于金刚石特 征峰的半高宽对应于金刚石膜的品质 ${ }^{[19]}$, 而其曲线 背底的强弱则直接反映了金刚石膜中缺陷密度的高 低 ${ }^{[20]}$, 因而由图 4 可以知道, MPCVD 法制备的金刚 石膜样品的品质优于 DC Arc Plasma Jet 法制备的金 刚石膜样品。

图 5 是两种不同方法制备的金刚石膜的紫外一可 见(UV-Visible)吸收光谱, 由图 5 可以看出, 在紫外一可 见光波段内, MPCVD 法制备的金刚石膜比 DC Arc Plasma Jet 法制备的金刚石膜的光吸收系数要低很 多。此外, 在 $270 \mathrm{~nm}$ 波长附近, DC Arc Plasma Jet 法制备的金刚石膜出现了明显的氮杂质吸收峰, 而 MPCVD 法制备的金刚石膜未出现明显的氮杂质吸 收峰。借助于样品在 $270 \mathrm{~nm}$ 波长处的吸收系数, 对 样品中氮杂质的含量进行估算, 采用的计算公式为 $0.56 \times 10^{-6} \times \Delta a^{[21]}$ 。其中, $\Delta a$ 参数的确定方法可以 通过图 5 中的插图得到解释。计算表明, MPCVD

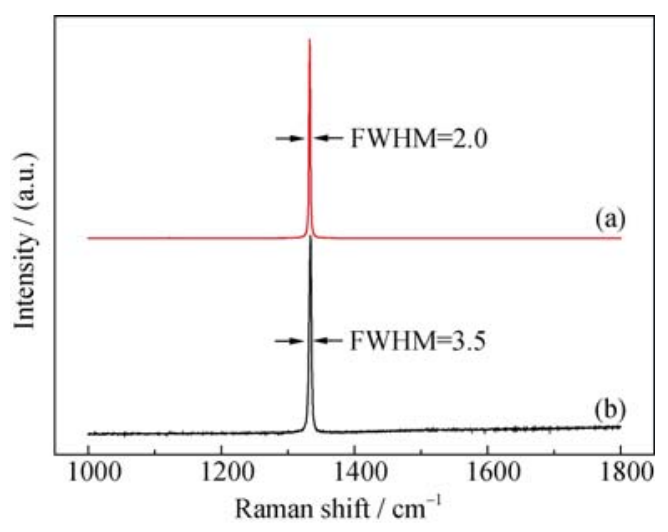

图 4 (a)MPCVD 法和(b) DC Arc Plasma Jet 法制备的金刚石 膜样品的 Raman 光谱图

Fig. 4 Raman spectra of the diamond films deposited by (a) MPCVD and (b) DC Arc Plasma Jet methods
法和 DC Arc Plasma Jet 法制备的金刚石膜样品中氮 杂质的含量分别约为 $0.7 \times 10^{-6}$ 和 $20.4 \times 10^{-6}$ 。

分别采用分体圆柱谐振腔式微波介电性能测试 装置对两种方法制备的金刚石膜进行了 $\mathrm{Ka}$ 波段的 微波介电性能测试。测试的结果如表 3 所示。由表 3 的数据可以看出, 在 $30 \mathrm{GHz}$ 频率附近, MPCVD法 制备的金刚石膜相对介电常数的测试值为 5.60, 而 DC Arc Plasma Jet 法制备的金刚石膜相对介电常数 的测试值为 5.77。这一测试结果与众多研究报道的 金刚石材料的介电常数值为 $5.5 \sim 5.8$ 相吻合 ${ }^{[22-23]}$ 。 此外, MPCVD 法制备的金刚石膜的微波损耗角正 切的测试值为 $1.38 \times 10^{-4}$, 而 DC Arc Plasma Jet 法制 备的金刚石膜的微波损耗角正切的测试值为 $7.49 \times 10^{-4}$ 。可见，相对介电常数还是微波损耗角正 切值, MPCVD 法制备的金刚石膜的测试值均低于 DC Arc Plasma Jet 法制备的金刚石膜。

GARRIDO 等 ${ }^{[21]}$ 的研究指出, 除常规 Raman 光 谱测试外，金刚石膜的介电特性表征也是一种判断 金刚石膜品质的好方法。随着金刚石膜品质的恶化, 其相对介电常数和微波介电损耗都将增大。由表 3 可见，MPCVD 法制备的金刚石膜拥有更低的相对 介电常数和损耗角正切, 即具有更高的品质。同时, 由前面对样品 Raman 光谱和紫外一可见光吸收谱的 测试结果可以看出, MPCVD 法制备的金刚石膜比 DC Arc Plasma Jet 法制备的金刚石膜具有更低的 Raman 谱半高宽值以及更低的氮杂质含量。由此可

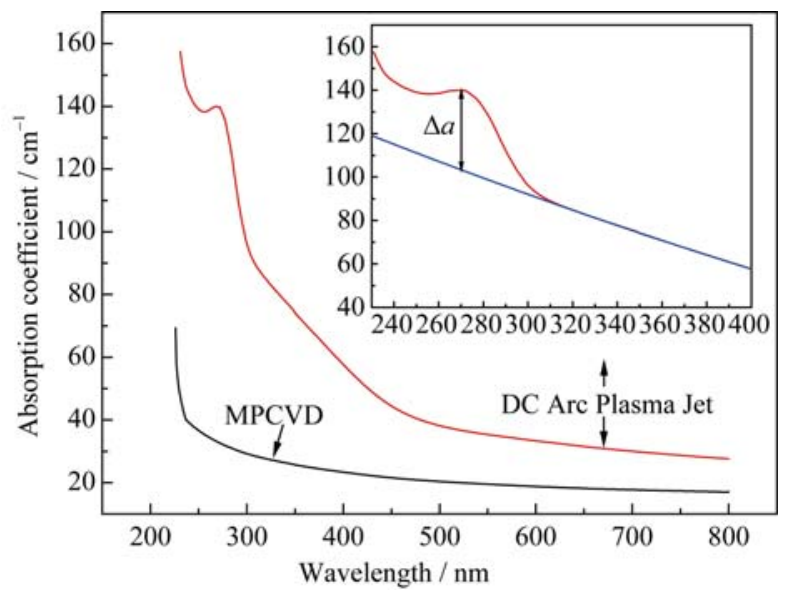

图 5 不同方法制备的金刚石膜样品的紫外-可见光吸收谱 Fig. 5 UV-visible absorption spectra of the diamond films deposited by different methods

表 3 两种不同方法制备的金刚石膜样品的微波介电性能测试结果

Table 3 Microwave dielectric properties of the diamond films deposited by different methods

\begin{tabular}{cccc}
\hline Deposition method & Frequency $/ \mathrm{GHz}$ & $\varepsilon_{\mathrm{r}}^{\prime}$ & $\tan \delta / \times 10^{-4}$ \\
\hline MPCVD & 29.280 & $5.60 \pm 0.07$ & $1.38 \pm 0.22$ \\
DC Arc Plasma Jet & 30.004 & $5.77 \pm 0.09$ & $7.49 \pm 0.66$ \\
\hline
\end{tabular}


以看出，上述两种不同方法制备的高品质金刚石膜 的介电性能表征结果与其 Raman 谱和紫外-可见光 吸收谱的表征结果是一致的, 拥有更小 Raman 谱半 高宽值以及更低的氮杂质含量的 MPCVD 法制备的 金刚石膜的微波介电性能更好。

DC Arc Plasma Jet 方法在制备金刚石膜时一般需 要消耗大量的气体, 而为了降低制备金刚石膜过程中 气体的消耗量, 在制备过程中采用了气体循环的工作 模式。此时, $\mathrm{CH}_{4} 、 \mathrm{H}_{2}$ 和 $\mathrm{Ar}$ 等工作气体被反复循环使 用 ${ }^{[17]}$ 。这种工作模式在大大降低了气体消耗量的同时, 必然会使金刚石膜中含有的杂质, 包括氮杂质的含量 有所增加, 而这必然导致金刚石膜微波介电性能在某 种程度上发生恶化。而相比而言, MPCVD 方法由于可 以提供极为洁净的沉积环境, 因而制备的金刚石膜的 杂质含量很低以及微波介电性能相对较好。

实验结果表明, 分体圆柱谐振腔式微波介电性 能测试装置可以满足金刚石膜这种低损耗薄膜材料 $\mathrm{Ka}$ 波段微波介电性能的测试需求。借助于所研制的 分体圆柱谐振腔式微波介电性能测试装置, 对两种 不同方法制备的高品质金刚石膜的介电性能进行了 初步的测试表征, 为进一步促进高品质金刚石膜材 料在微波电真空器件领域中的应用打下了基础。

\section{4 结论}

实验建立了一种分体圆柱谐振腔式微波介电性能 测试装置, 其 $\mathrm{TE}_{011}$ 模式的空腔谐振频率 $f_{011}$ 为 $35 \mathrm{GHz}$, 可用于低损耗薄膜类样品在 $\mathrm{Ka}$ 波段下的微波介电 性能测试。借助于对不同直径的蓝宝石单晶样品的 测试, 对分体圆柱谐振腔式微波介电性能测试装置 的测试能力进行了验证。测试所得的所有蓝宝石单 晶样品的相对介电常数和损耗角正切值均处于合理 范围内, 显示分体圆柱谐振腔式微波介电性能测试 系统具有良好的测试稳定性。

对高功率 MPCVD 和 DC Arc Plasma Jet 两种不 同方法制备的高品质金刚石膜进行了 $\mathrm{Ka}$ 波段微波 介电性能的测试。测试结果显示, 高功率 MPCVD 法制备的金刚石膜的相对介电常数和微波损耗均低 于 DC Arc Plasma Jet 法制备的金刚石膜, 这与 Raman 光谱、紫外-可见光谱表征的 MPCVD 法制备 的金刚石膜的品质优于 DC Arc Plasma Jet 法制备的 金刚石膜的结果相吻合。

\section{参考文献:}

[1] LI X Y, GUO H, LIU Z, et al. Applications of CVD diamond film to vacuum electronic devices. Vacuum Electronics, 2009, 4: 27-31.

[2] NUSSNTARA H, SETIAWAN A, CHAIRUNNISA, et al. Investigation of dielectric-lined for transmission loss reduction of optical waveguide. Procedia Technology, 2013, 11: 1117-1121.

[3] KUMAR A, KUMAR N, SINGH U, et al. Towards a $1 \mathrm{MW}, 170 \mathrm{GHz}$ gyrotron design for fusion application. Infrared Phys. Technol., 2013, 57: 1-7.

[4] MANFRED T. Progress in gyrotron development. Fusion Eng. Des., 2003, 66-68: 69-90.

[5] TAKAHASHI K, KAJIWARA K, ODA Y, et al. High power millimeter wave experiment of torus diamond window prototype for ITER EC H\&CD system. Fusion Engineering and Design, 2013, 88(2): 85-93.

[6] SCHEURING A, PROBST P, STOCKHAUSEN A, et al. Dielectric RF Properties of CVD Diamond Disks from Sub-mm Wave to $\mathrm{THz}$ Frequencies. 35th Int. Conf. on Infrared, Millimeter, and THz Waves, Rome, 2010: 1-2.

[7] SHUKLA B K, BABU R, KUSHWAH M, et al. High-power test of chemical vapor deposited diamond window for an ECRH system in SST-1. IEEE Transactions on Plasma Science, 2013, 41(7): 1794-1798.

[8] SCHERER T A, STRAUSS D, TORGE M, et al. Investigations of Dielectric RF Properties of Ultra Low Loss CVD Diamond Disks for Fusion Applications. 34th Int. Conf. on Infrared, Millimeter, and THz Waves, Tucson, 2009: 1-2.

[9] YANG X, BORIE E, DAMMERTZ G, et al. The influence of window parameters on the transmission characterisitics of millimeter waves. Int. J. Infrared Milli. Waves, 2003, 24(11): 1805-1813.

[10] POLYAKOV V I, RUKOVISHNIKOV A I, GARIN B M, et al. Electrically active defects, conductivity, and millimeter wave dielectric loss in CVD diamonds. Diamond Relat. Mater, 2005, 14(3-7): 604-607.

[11] XIN H W, ZHANG Z M, LING X, et al. Composite diamond films with smooth surface and the structural influence on dielectric properties. Diamond Relat. Mater., 2002, 11(2): 228-233.

[12] LU F X, ZHANG H D, TONG Y M, et al. Dielectric property of thick freestanding diamond films by high power arcjet operating at gas recycling mode. Diamond Relat. Mater, 2004, 13(9): 1714-1718.

[13] JANEZIC M D, KUESTER E F, BAKER-JARVIS J. Broadband complex permittivity measurements of dielectric substrates using a split-cylinder resonator. 2004 IEEE MIT-S Digest, 2004, $1817-1820$.

[14] JANEZIC M D. Nondestructive Relative Permittivity and Loss Tangent Measurements Using a Split-cylinder Resonator. Denver: University of Colorado, 2003. 
[15] SHAPIRO M A, MOELLER C P, TEMKIN R J, et al. Electromagnetic analysis and cold test of a distributed window for a high power gyrotron. Int. J. Infrared and Millimeter Waves, 1999, 20(4): $533-542$

[16] SILVA F, HASSOUNI K, BONNIN X, et al. Microwave engineering of plasma-assisted CVD reactors for diamond deposition. $J$. Phys.: Condens. Matter, 2009, 21(36): 364202.

[17] LU F X, LI C M, TONG Y M, et al. Application of high power DC arc plasma for mass production of high quality freestanding diamond films and diamond film coated cutting tools. Mater. Sci. Forum, 2010, 654-656: 1694-1699.

[18] SU J J, LI Y F, DING M H, et al. A dome-shaped cavity type microwave plasma chemical vapor deposition reactor for diamond films deposition. Vacuum, 2014, 107: 51-55.
[19] PRAWER S, NEMANICH R J. Raman spectroscopy of diamond and doped diamond. Philos. Trans. R. Soc. London, Ser. A, 2004, 362: $2537-2565$.

[20] GONON P, GHEERAERT E, DENEUVILLE A, et al. Raman study of diamond films deposited by MPCVD: effect of the substrate position. Thin Solid Films, 1995, 256(1-2): 13-22.

[21] GARRIDO O S, ALEIXANDRE C G, OLIAS J S, et al. Dielectric and Raman spectroscopy of MWCVD diamond thin films. J. Mater. Sci. - Mater. Electron., 1996, 7: 297-303.

[22] IBARRA A, GONZÁLEZ M, VILA R, et al. Wide frequency dielectric properties of CVD diamond. Diamond Relat. Mater., 1997, 6: $856-859$

[23] XU P, YANG J, QIU T. The progress of output window materials. Bulletin of the Chinese Ceramic Society, 2007, 26(6): 1160-1164. 